\title{
On the spin-to-orbit momentum conversion operated by electric field in optically active $\mathrm{Bi}_{12} \mathrm{GeO}_{20}$ crystals
}

\author{
Vasylkiv Yu., Krupych O., Skab I. and Vlokh R. \\ Institute of Physical Optics, 23 Dragomanov St., 79005 Lviv, Ukraine, \\ vlokh@ifo.lviv.ua
}

Received: 07.10.2011

\begin{abstract}
An optical conversion of spin angular momentum to orbital angular momentum (SAM-to-OAM) that appears in $\mathrm{Bi}_{12} \mathrm{GeO}_{20}$ crystals under the effect of conically shaped external electric field due to a Pockels effect has been studied both experimentally and theoretically. We have revealed the appearance of a doughnut mode and an optical vortex in the system consisting of a right-handed circular polariser, a sample subjected to conical electric field, and a left-handed circular analyser. It has been found that the presence of natural optical activity leads to notable decrease in the efficiency of the SAM-to-OAM conversion.
\end{abstract}

Keywords: optical vortex, natural optical activity, Pockels effect, $\mathrm{Bi}_{12} \mathrm{GeO}_{20}$ crystals

PACS: 78.20.Jq, 42.50.Tx, 78.20.Ek

UDC: $535.542,535.56$

\section{Introduction}

The interest to real-time operation by an orbital angular momentum (OAM) of light beams is caused by promising applications of this phenomenon in quantum computing, cryptography and quantum teleportation [1-3]. The information can be encoded by multiplying a number of states, since a photon can carry an arbitrarily large amount of information distributed over its spin and orbital quantum states [4]. In addition, the OAM operation enables increasing number of parameters by which optical beams could be modulated. More specifically, any information transferred from a driving signal can be encoded not only into, e.g., phase, intensity, polarisation or frequency of light, but also into its OAM. As a result, development of direct methods for the OAM operation should lead to novel possibilities for applications of quantum properties of photons. However, this is hindered by the problem of finding the appropriate material media for real-time conversion of spin angular momentum (SAM) to OAM (abbreviated hereafter as a SAMto-OAM conversion).

Recently we have shown that the OAM can be efficiently operated using twisting of crystals [5-9] or via a Pockels effect occurring in case when a conically shaped spatial distribution of external electric field is created in a crystal $[10,11]$. The last method allows for direct operation of the OAM by electrical driving signals. We have demonstrated experimentally that an optical vortex appears whenever a conically shaped electric field is applied to $\mathrm{LiNbO}_{3}$ crystals. It has also been shown that, under crystal torsion, a pure screw dislocation of the phase front appears in the crystalline materials belonging to the point groups of symmetry $\mathrm{m} 3 \mathrm{~m}, \mathrm{~m} 3,23,432, \overline{4} 3 \mathrm{~m}, 3 \mathrm{~m}$, $\overline{3} \mathrm{~m}, 32,3$, and $\overline{3}$. We should also have the same effect when a conically shaped electric field is applied to the crystals of the groups $3 \mathrm{~m}, 32,3, \overline{6}$, and $\overline{6} \mathrm{~m} 2,23$, and $\overline{4} 3 \mathrm{~m}$. 
Notice that some of the symmetry groups mentioned above (namely, 23, 432, 32, and 3) permit the existence of a natural optical activity. Hence a following reasonable question arises: how does the natural optical activity affect the efficiency of the SAM-to-OAM conversion, for instance if the vortex is being induced by the electric field via the Pockels effect? We are to remind in this respect that the crystals permitting the appearance of the Pockels effect, on the one hand, and the natural optical activity, on the other hand, belong almost to the same point symmetry groups. The linear electrooptic effect and the natural optical activity coexist at least in the groups $23,432,32$, and 3 . The problem can be solved, for example, while analysing the simplest case of cubic crystals as materials for operating the OAM electrooptically. These crystals are initially optically isotropic. The condition of simultaneous availability of optical isotropy in all the directions of light propagation, a notable natural optical activity and high enough electrooptic coefficients are well met for $\mathrm{Bi}_{12} \mathrm{GeO}_{20}$ crystals which represent an efficient electrooptic material.

The aim of the present work is experimental and theoretical studies of electrooptically operated SAM-to-OAM conversion in $\mathrm{Bi}_{12} \mathrm{GeO}_{20}$ crystals in the presence of optical rotation.

\section{Experimental results and discussion}

$\mathrm{Bi}_{12} \mathrm{GeO}_{20}$ is a well-known electrooptic crystal with the Pockels coefficient $r_{41}=3.5 \mathrm{pm} / \mathrm{V}$ and the refractive index $n=2.55$ for the light wavelength $\lambda=632.8 \mathrm{~nm}$ [12]. The crystal possesses a notable optical activity (the specific optical rotation is equal to $\delta=20 \mathrm{deg} / \mathrm{mm}$ [12]). Besides, the crystal is photorefractive and so can be used for optical storage (see, e.g., [13]). The experimental techniques employed while studying the optical indicatrix rotation and observing the helical mode have been described in detail in our recent works $[10,11]$. Here we will briefly remind only the main relations that describe the Pockels effect appearing under the action of conically shaped electric field.

The relation describing the Pockels effect is as follows:

$$
\Delta B_{i}=r_{i j} E_{j}
$$

where $\Delta B_{i}$ denote the increments of optical impermeability tensor, $E_{j}$ the electric field components, and $r_{i j}$ the electrooptic tensor. Eq. (1) written in the eigen (crystallographic) coordinate system for the $\mathrm{Bi}_{12} \mathrm{GeO}_{20}$ crystals belonging to the point symmetry group 23 may be presented in the form

\begin{tabular}{c|ccc} 
& $E_{1}$ & $E_{2}$ & $E_{3}$ \\
\hline$\Delta B_{1}$ & 0 & 0 & 0 \\
$\Delta B_{2}$ & 0 & 0 & 0 \\
$\Delta B_{3}$ & 0 & 0 & 0 \\
$\Delta B_{4}$ & $r_{41}$ & 0 & 0 \\
$\Delta B_{5}$ & 0 & $r_{41}$ & 0 \\
$\Delta B_{6}$ & 0 & 0 & $r_{41}$
\end{tabular}

As shown in the work [10], a unique condition for producing the pure screw dislocation of the phase front in cubic crystals appears when the conically shaped electric field is formed along all of $<111>$ directions and the optical wave propagates along the same directions (see $[10,11]$ ). Then it would be convenient to rewrite the matrix given by Eq. (2) in the coordinate system whose $Z^{\prime}$ axis coincides with the direction [111]. In this manner one can arrive at the following nonzero tensor components: 


$$
r_{12}^{\prime}=r_{21}^{\prime}=-r_{13}^{\prime}=-r_{23}^{\prime}=-r_{11}^{\prime}=-r_{22}^{\prime}=r_{33}^{\prime} / 2=-r_{42}^{\prime}=-r_{51}^{\prime}=r_{61}^{\prime}=r_{62}^{\prime}=r_{41} / \sqrt{3} .
$$

The equation for the cross section of the Fresnell ellipsoid reads as

$$
\left(B_{1}+r_{11}^{\prime}\left(E_{X^{\prime}}-E_{Y^{\prime}}+E_{Z^{\prime}}\right)\right) X^{\prime^{2}}+\left(B_{1}-r_{11}^{\prime}\left(E_{X^{\prime}}-E_{Y^{\prime}}-E_{Z^{\prime}}\right)\right) Y^{\prime 2}-2 r_{11}^{\prime}\left(E_{X^{\prime}}+E_{Y^{\prime}}\right) X^{\prime} Y^{\prime}=1,
$$

while the spatial distributions of the optical birefringence and the angle of optical indicatrix rotation are given respectively by the relations

$$
\begin{aligned}
& \Delta n_{X^{\prime} Y^{\prime}}=\sqrt{\frac{2}{3}} n^{3} r_{41} \sqrt{E_{X^{\prime}}^{2}+E_{Y^{\prime}}^{2}}=\sqrt{\frac{2}{3}} n^{3} r_{41} E_{0} \frac{\rho^{\prime}}{\rho^{2}} Z^{\prime}, \\
& \tan 2 \zeta_{Z^{\prime}}=-\frac{\left(X^{\prime}+Y^{\prime}\right)}{\left(X^{\prime}-Y^{\prime}\right)}=-\frac{\cos \varphi+\sin \varphi}{\cos \varphi-\sin \varphi}=\tan \left(\frac{3 \pi}{4}-\varphi\right) \quad\left(\text { or } \zeta_{Z^{\prime}}=\frac{3 \pi}{8}-\frac{\varphi}{2}\right) .
\end{aligned}
$$

Here $E_{0}=U / d, U$ stands for the electric voltage applied, $d$ the thickness of a crystalline plate, $E_{X^{\prime}}=E_{0} \frac{Z^{\prime}}{\rho^{2}} X^{\prime}, E_{Y^{\prime}}=E_{0} \frac{Z^{\prime}}{\rho^{2}} Y^{\prime}$, and the polar coordinate system has been introduced via standard relations $X^{\prime}=\rho^{\prime} \cos \varphi$ and $Y^{\prime}=\rho^{\prime} \sin \varphi$ (see Fig. 1).

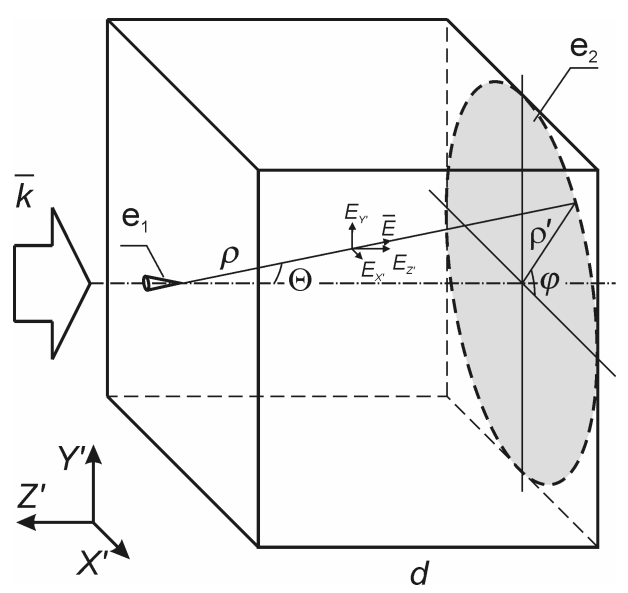

Fig. 1. Schematic representation of a crystalline plate with circular electrodes $e_{1}$ and $e_{2}$, and a conical spatial distribution of electric field produced by these electrodes $(R=8 \mathrm{~mm}$ is taken for the radius of the larger electrode and $d=6 \mathrm{~mm}$ for the sample thickness).

The experimental spatial distribution of the angle of optical indicatrix rotation induced by the conical electric field in the $\mathrm{Bi}_{12} \mathrm{GeO}_{20}$ crystals is presented in Fig. 2. Here the undefined value of the optical indicatrix rotation angle corresponds to the central part of the map (i.e., to the coordinates $X^{\prime}=-0.53 \mathrm{~mm}$ and $Y^{\prime}=-0.34 \mathrm{~mm}$ ). The rotation angle gradually changes its value from $0 \mathrm{deg}$ to $180 \mathrm{deg}$ when the tracing angle $\varphi$ is changing from $0 \mathrm{deg}$ to $360 \mathrm{deg}$, due to rotation around the origin. Obviously, the orientation of azimuth of the polarisation ellipse for the light emerging from the sample follows the orientation of the principal axes of optical indicatrix, i.e. it rotates by the angle $\beta=180 \mathrm{deg}$ when the tracing angle changes its value by $\Delta \varphi=360 \mathrm{deg}$. The change in the azimuth orientation of the polarisation ellipse equal to $180 \mathrm{deg}$ corresponds to the change by $360 \mathrm{deg}$ for the phase of the light wave, as a consequence of the relation $\beta=\pi d \Delta n / \lambda=\Delta \Gamma / 2$. Thus, the screw-like spatial distribution of the optical indicatrix rotation observed around the vortex core is a strong argument for appearance of dislocation of the phase 
front and, hence, a helical mode. On the other hand, the map of the optical indicatrix orientation in the $X^{\prime} Y^{\prime}$ plane is characterised by a topological defect with the strength equal to $1 / 2$ (the optical indicatrix rotation angle is twice as smaller when compared with the tracing angle), so that the vortex transferred by the outgoing beam should be characterised by the OAM equal to unity.

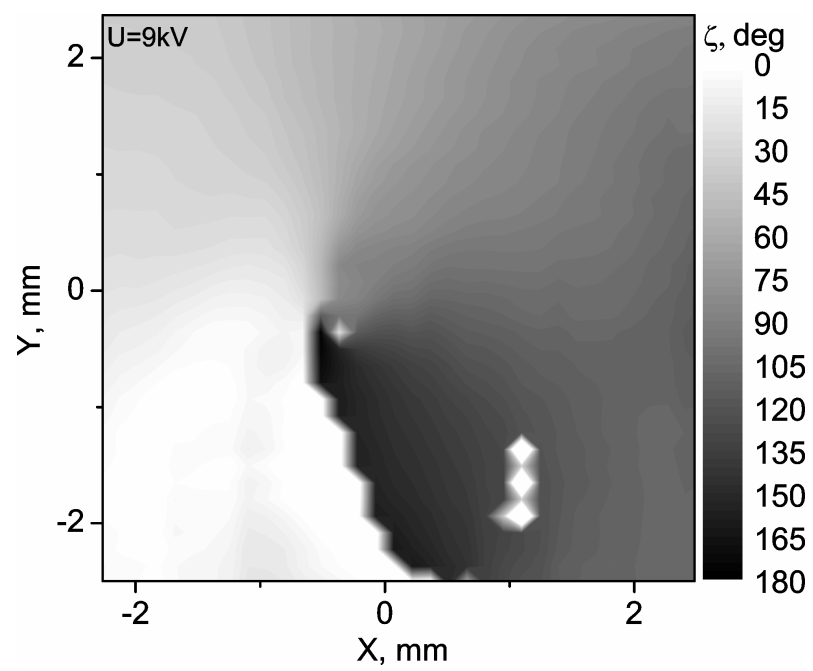

Fig. 2. Experimental distribution of optical indicatrix rotation angle induced in the $\mathrm{Bi}_{12} \mathrm{GeO}_{20}$ crystals by the conically shaped electric field (the electric voltage $U=9.0 \mathrm{kV}$ and $\lambda=632.8 \mathrm{~nm}$ ).
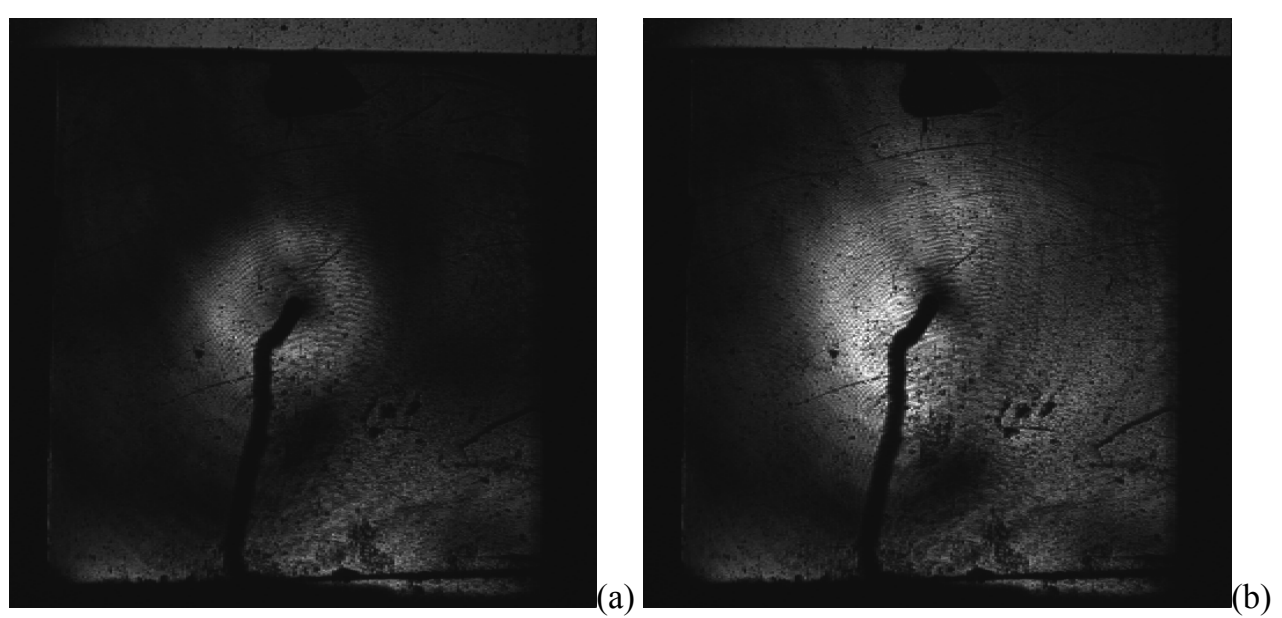

Fig. 3. The view of a helical mode induced in the $\mathrm{Bi}_{12} \mathrm{GeO}_{20}$ crystals by the electric voltages $6 \mathrm{kV}(\mathrm{a})$ and $9 \mathrm{kV}(\mathrm{b})$.

The intensity distribution observed for the beam emerging from the optical system that containes sequentially a right-handed circular polariser, a crystalline sample under conical electric field, and a left-handed circular analyser is shown in Fig. 3. The distribution given in Fig. 3 is typical for the optical vortex: a dark central part is surrounded by a bright ring. However, comparatively long-term application of the voltage combined with illumination of the sample by optical radiation ( $\lambda=632.8 \mathrm{~nm}$ ) leads to smearing of the doughnut mode, the effect clearly visible in Fig. 3b. Most probably, this process is caused by a photorefraction effect accompanying spatial redistribution of photo-induced charges. 
The $X^{\prime} Y^{\prime}$ intensity distribution for the outgoing beam can be calculated using Jones matrices. Since the birefringence depends on all the coordinates $X^{\prime}, Y^{\prime}$ and $Z^{\prime}$, it is convenient to divide our sample into $n$ homogeneous layers perpendicular to the $Z^{\prime}$ direction. In its turn, each of the layers is divided into $k \times l$ homogeneous elementary cells in the $X^{\prime} Y^{\prime}$ plane. In practice, we have used a division given by $k=100, l=100$, and $n_{\max }=1 \div 100$. Now one can use a standard Jones matrix approach for simulating the electrically induced phase difference. Here we assume that the linear birefringence is not induced by the electric field in the spatial domain lying out of the electric force lines limited by a cone (see Fig. 1). Then the sample should be divided into the two parts: the first one (inside the cone) is characterised by an elliptical birefringence, whereas the second (out of the region limited by the cone) reveals the pure optical activity and so a circular birefringence only. The resulting Jones matrix for the first part of the sample can be written as

$$
J_{\text {ellipt }}^{i j}=\prod_{n=1}^{n_{\max }} J_{n}^{i j}
$$

where

$$
J_{n}^{i j}=\left|\begin{array}{cc}
\cos \frac{\Delta \Gamma_{n}^{i j}}{2}+i \sin \frac{\Delta \Gamma_{n}^{i j}}{2} \cos 2 \kappa_{n}^{i j} \cos 2 \zeta_{n}^{i j} & \sin \frac{\Delta \Gamma_{n}^{i j}}{2} \sin 2 \kappa_{n}^{i j}+i \sin \frac{\Delta \Gamma_{n}^{i j}}{2} \cos 2 \kappa_{n}^{i j} \sin 2 \zeta_{n}^{i j} \\
-\sin \frac{\Delta \Gamma_{n}^{i j}}{2} \sin 2 \kappa_{n}^{i j}+i \sin \frac{\Delta \Gamma_{n}^{i j}}{2} \cos 2 \kappa_{n}^{i j} \sin 2 \zeta_{n Z}^{i j} & \cos \frac{\Delta \Gamma_{n}^{i j}}{2}-i \sin \frac{\Delta \Gamma_{n}^{i j}}{2} \cos 2 \kappa_{n}^{i j} \cos 2 \zeta_{n Z}^{i j}
\end{array}\right| .
$$

The phase difference for each elementary cell is equal to

$$
\Delta \Gamma_{n}^{i j}=\frac{2 \pi d_{n}^{i j}}{\lambda}\left\{\left(\sqrt{\frac{2}{3}} n^{3} r_{41} E_{0} \frac{\sqrt{X_{i}^{\prime 2}+Y_{j}^{\prime 2}}}{X_{i}^{\prime 2}+Y_{j}^{\prime 2}+Z_{n}^{\prime 2}} Z_{n}^{\prime}\right)^{2}+\left(\frac{\delta \lambda}{\pi}\right)^{2}\right\}^{1 / 2},
$$

while the ellipticity of the eigenwaves is determined by

$$
\kappa_{n}^{i j}=-\arctan \frac{-\Delta n_{X^{\prime} Y^{\prime}}+\sqrt{\Delta n_{X^{\prime} Y^{\prime}}^{2}+g_{3}^{2}}}{g_{3}} .
$$

Here $\Delta \Gamma_{n}^{i j}$ and $\zeta_{n Z^{\prime}}^{i j}=\frac{1}{2} \arctan \frac{X_{i}^{\prime}+Y^{\prime}{ }_{j}}{X_{i}^{\prime}-Y^{\prime}{ }_{j}}$ represent respectively the phase difference and the angle of optical indicatrix rotation within the elementary cells, $g_{3}=\delta \lambda n / \pi$ is the gyration tensor component, and $d_{n}^{i j}$ the thickness of the cell along the direction of light propagation.

The Jones matrix $J_{\text {circ }}^{i j}$ for the second part of the sample is determined by Eq. (8) under the conditions $\Delta \Gamma^{i j}=\frac{2 \delta d \sqrt{X_{i}^{\prime 2}+Y_{j}^{\prime 2}}}{R}, \kappa^{i j}=\frac{\pi}{4}, \zeta^{i j}=0$. Thus, the resulting Jones matrix becomes as

$$
J^{i j}=J_{\text {ellipt }}^{i j} \times J_{\text {circ }}^{i j},
$$

while the resulting phase difference may be written as

$$
\Delta \Gamma^{i j}=2 \arccos \left(\operatorname{Re}\left(J_{11}^{i j}\right)\right),
$$

with $J_{11}^{i j}$ being the component of the Jones matrix.

As a result, we have

$$
\left(\begin{array}{c}
E_{1}^{i j} \\
E_{2}^{i j}
\end{array}\right)=J^{A} J^{Q W P-} J^{i j} J^{Q W P+}\left(\begin{array}{c}
E_{1} \\
E_{2}
\end{array}\right),
$$


where

$$
\begin{aligned}
& E_{1}=1, \quad E_{2}=0, \quad J^{A}=\left(\begin{array}{ll}
0 & 0 \\
0 & 1
\end{array}\right), \\
& J^{Q W P-}=\left(\begin{array}{ll}
\frac{1}{\sqrt{2}} e^{i \frac{\pi}{4}} & \frac{1}{\sqrt{2}} e^{-i \frac{\pi}{4}} \\
\frac{1}{\sqrt{2}} e^{-i \frac{\pi}{4}} & \frac{1}{\sqrt{2}} e^{i \frac{\pi}{4}}
\end{array}\right), \quad J^{Q W P+}=\left(\begin{array}{ll}
\frac{1}{\sqrt{2}} e^{-i \frac{\pi}{4}} & \frac{1}{\sqrt{2}} e^{i \frac{\pi}{4}} \\
\frac{1}{\sqrt{2}} e^{i \frac{\pi}{4}} & \frac{1}{\sqrt{2}} e^{-i \frac{\pi}{4}}
\end{array}\right) .
\end{aligned}
$$

Here $E_{1}, E_{2}$ and $E_{1}^{i j}, E_{2}^{i j}$ are the components of the input and output Jones vectors, respectively, and $J^{Q W P_{-}}, J^{Q W P+}$ and $J^{A}$ the Jones matrices of the quarter wave plates rotated by $\pm 90 \mathrm{deg}$ and the analyser, respectively. The resulting intensity for each of the elementary beams is determined by the relation

$$
\left(I^{i j}\right)_{\text {lefthanded }}^{\text {out }}=\left(\begin{array}{c}
E_{1}^{i j} \\
E_{2}^{i j}
\end{array}\right)\left(\begin{array}{c}
E_{1}^{i j^{*}} \\
E_{2}^{i j^{*}}
\end{array}\right) .
$$

One can characterise the incident beam as a nearly plane wave, with the SAM equal to $S^{i n c}=-\hbar$. Then the electric field of the emergent light is as follows:

$$
E^{\text {out }}\left(\rho^{\prime}, \varphi\right)=E_{A} \cos \frac{\Delta \Gamma\left(\rho^{\prime}\right)}{2}\left[\begin{array}{c}
1 \\
\pm i
\end{array}\right]+i E_{A} \sin \frac{\Delta \Gamma\left(\rho^{\prime}\right)}{2} e^{ \pm i 2 q \varphi \pm i 2 \alpha_{0}}\left[\begin{array}{c}
1 \\
\mp i
\end{array}\right]
$$

where $2 q=m=1$ is the helicity number and $E_{A}$ the wave amplitude. The first term in the r. h. s. of Eq. (16) describes the plane wave with the same SAM as in the incident one, while the second term corresponds to the doughnut mode possessing the helical wave front that carries some OAM (see [14-17]). Since the angular momentum must be conserved, one can write the following relation for the SAM-to-OAM conversion:

$$
J^{\text {inc }}=J^{\text {out }}+M,
$$

where $J^{i n c}=S^{i n c}=-\hbar$ is the total angular momentum of the incident photon, $J^{\text {out }}=L^{\text {out }}+S^{\text {out }}=-2 q \hbar+\hbar=0$ the total angular momentum of the emergent photon ( $\left.S^{\text {out }}=+\hbar, L^{\text {out }}=-2 q \hbar\right)$, and $L^{\text {out }}$ the OAM of the latter. The mechanical angular momentum transferred to the crystalline sample due to the Beth effect [18] is therefore equal to $M=-\hbar$. Notice that the above relations which reflect conservation of the angular momentum have been written under the condition $\Delta \Gamma=\pi$, though the phase difference can really depend on the module $\rho^{\prime}$. In this case one should take into account that the plane wave described by the first term in the r. h. s. of Eq. (16), with the SAM equal to $-\hbar$, also emerges from the sample. Then the efficiency of the SAM-to-OAM conversion should be defined by the ratio

$$
\eta=\frac{I_{\text {lefthanded }}^{\text {out }}}{I_{\text {righthanded }}^{\text {inc }}},
$$

where $I_{\text {righthanded }}^{\text {inc }}$ is the intensity of the right-handed incident wave and $I_{\text {lefthanded }}^{\text {out }}$ that of the lefthanded outgoing wave. 
We have found that the efficiency of the SAM-to-OAM conversion calculated on the basis of Eq. (18) and the appropriate literature data for the electrooptic coefficient, refractive index and the optical rotation, is equal to $0.6 \%$ at the voltage of $6 \mathrm{kV}$ (see Fig. 4a). It is worthwhile that, at the same conditions, the efficiency of the SAM-to-OAM conversion would be equal to $6.7 \%$ without accounting of the natural optical activity. This is an order of magnitude higher than the figure mentioned before (see Fig. $4 \mathrm{~b}$ ). The corresponding spatial distributions of the phase differences are presented in Fig. 5.
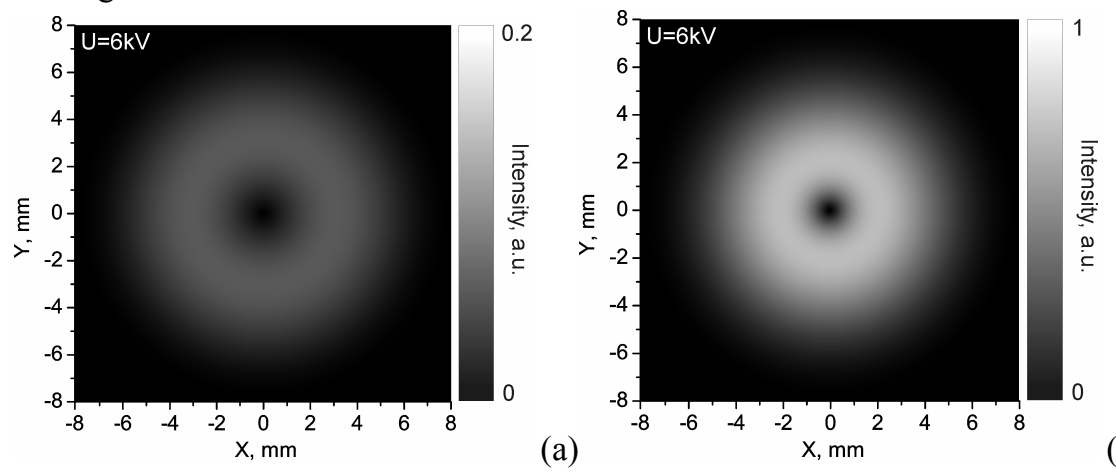

(b)

Fig. 4. Calculated intensity distributions of doughnut mode appearing in the $\mathrm{Bi}_{12} \mathrm{GeO}_{20}$ crystals under the voltage $6 \mathrm{kV}$ : (a) with taking the natural optical activity into consideration, and (b) with no consideration of the effect $(\lambda=632.8 \mathrm{~nm})$.
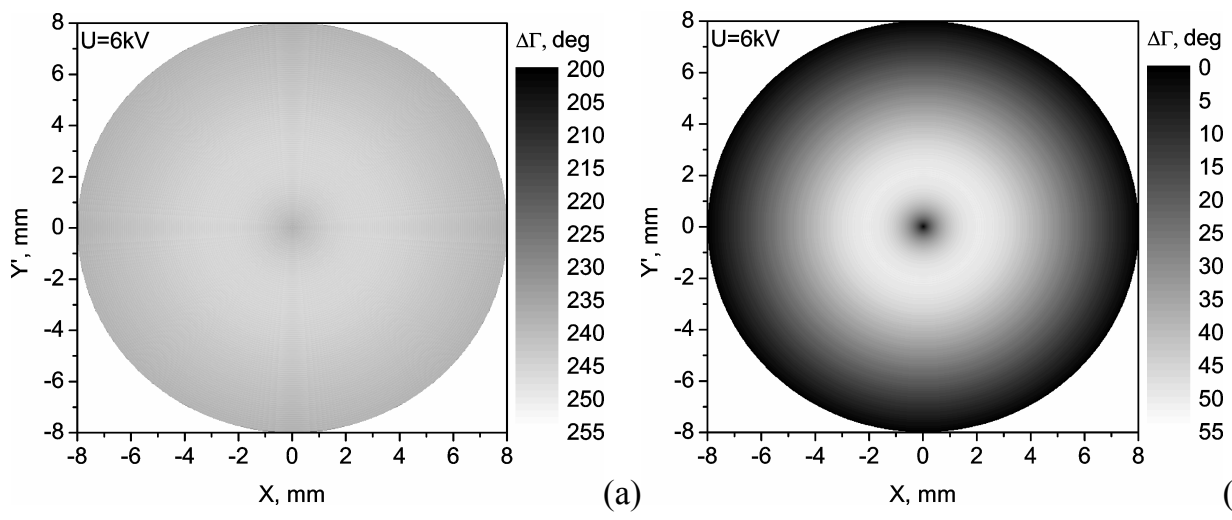

Fig. 5. Calculated maps of phase difference induced in the $\mathrm{Bi}_{12} \mathrm{GeO}_{20}$ crystals by the voltage $6 \mathrm{kV}$ : with taking the natural optical activity into consideration, and (b) with no consideration of the effect $(\lambda=632.8 \mathrm{~nm})$.

Hence, the natural optical activity should lead to essential decrease in the efficiency of the SAM-to-OAM conversion. Besides, the efficiency of the SAM-to-OAM conversion can depend upon the electric voltage applied and the sample thickness along the $Z^{\prime}$ direction. The dependences of the efficiency on the sample thickness simulated for the $\mathrm{Bi}_{12} \mathrm{GeO}_{20}$ crystals at different electric voltages are displayed in Fig. 6.

As seen from Fig. 6, the maximum efficiency is achieved for the sample thickness of $d=2.5 \mathrm{~mm}$ if the optical rotation is present and $d=5.0 \mathrm{~mm}$ if it is absent. Notice that the sample thickness corresponding to the maximum efficiency of the SAM-to-OAM conversion is almost independent of the electric voltage. At the same time, the efficiency $\eta$ itself does depend on the voltage applied. For the voltages as high as $12 \mathrm{kV}$, it reaches the value of $5.3 \%$ in the presence of optical rotation, and $22.7 \%$ in the absence of the effect. 


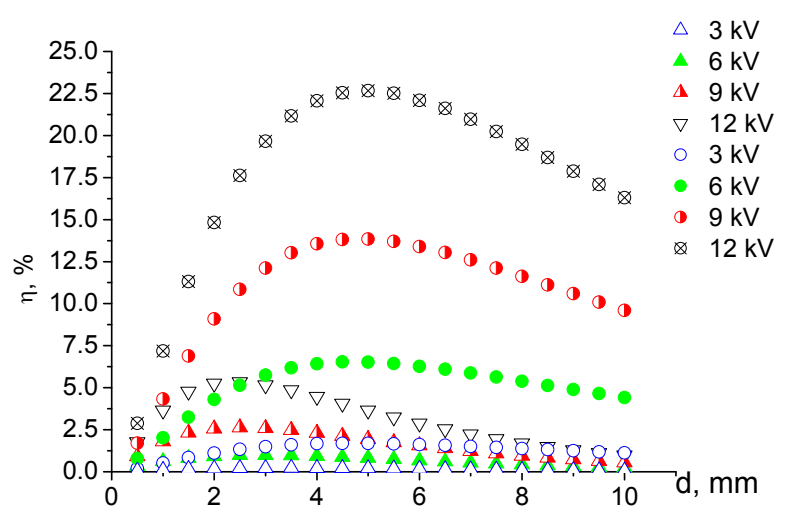

Fig. 6. Calculated dependences of efficiency of the SAM-to-OAM conversion occurred in the $\mathrm{Bi}_{12} \mathrm{GeO}_{20}$ crystals on the sample thickness at different voltages applied: triangles and circles correspond respectively to accounting for the natural optical activity and disregarding the effect $(\lambda=632.8 \mathrm{~nm}$ and $R=8 \mathrm{~mm})$.

\section{Conclusions}

In the present work we have revealed experimentally the SAM-to-OAM conversion in $\mathrm{Bi}_{12} \mathrm{GeO}_{20}$ crystals appearing under the conically shaped electric field due to the Pockels effect. It has been shown that the vortex of a unit charge appears in the optical system that includes the right-handed circular polariser, the crystalline sample under the conical electric field, and the left-handed circular analyser. We have found that the natural optical activity imposes decreasing efficiency of the SAM-to-OAM conversion. In case of the $\mathrm{Bi}_{12} \mathrm{GeO}_{20}$ crystals, the latter decreases almost four times for the voltage $12 \mathrm{kV}$.

Besides, we emphasise that the spatial intensity distributions of the type presented in Fig. 4 can be used in order to measure the electrooptic coefficient $r_{41}$, whenever all the other parameters involved in Eq. (9) are known in advance. The procedure should be similar to that used for measuring the piezooptic coefficients under crystal torsion, as described in the study [19].

\section{References}

1. DiVincenzo D P, 1995. Quantum computation. Science. 270: 255-261.

2. Kilin S Ya, 1999. Quantum information. Sov. Phys. Uspekhi. 42: 435-452.

3. Boschi D, Branca S, De Martini F, Hardy L and Popescu S, 1998. Experimental realization of teleporting an unknown pure quantum state via dual classical and Einstein-Podolsky-Rosen channels. Phys. Rev. Lett. 80: 1121-1125.

4. Molina-Terriza G, Torres J P and Torner L, 2001. Management of the angular momentum of light: preparation of photons in multidimensional vector states of angular momentum. Phys. Rev. Lett. 88: 013601.

5. Skab I, Vasylkiv Yu, Savaryn V and Vlokh R, 2011. Optical anisotropy induced by torsion stresses in $\mathrm{LiNbO}_{3}$ crystals: appearance of an optical vortex. J. Opt. Soc. Amer. A. 28: 633640.

6. Skab I, Vasylkiv Yu, Zapeka B, Savaryn V and Vlokh R, 2011. Appearance of singularities of optical fields under torsion of crystals containing threefold symmetry axes. J. Opt. Soc. Amer. A. 28: 1331-1340.

7. Vasylkiv Yu, Savaryn V, Smaga I, Skab I and Vlokh R, 2010. Determination of piezooptic coefficient $\pi_{14}$ of $\mathrm{LiNbO}_{3}$ crystals under torsion loading. Ukr. J. Phys. Opt. 11: 156-164. 
8. Skab I, Vasylkiv Yu, Savaryn V and Vlokh R, 2010. Relations for optical indicatrix parameters in the conditions of crystal torsion. Ukr. J. Phys. Opt. 11: 193-240.

9. Skab I, Vasylkiv Yu, Smaga I, Savaryn V and Vlokh R, 2011. On the method for measuring piezooptic coefficients $\pi_{25}$ and $\pi_{14}$ in the crystals belonging to point symmetry groups 3 and $\overline{3}$. Ukr. J. Phys. Opt. 12: 28-35.

10. Skab I, Vasylkiv Yu, Smaga I and Vlokh R, 2011. Spin-to-orbital momentum conversion via electrooptic Pockels effect in crystals. Phys. Rev. A 84: 043815.

11. Skab I P, Vasylkiv Yu V and Vlokh R O, 2011. On the possibility of electrooptic operation by orbital angular momentum of light beams via Pockels effect in crystals. Ukr. J. Phys. Opt. 12: 127-136.

12. http://www.almazoptics.com/BGO-BSO-BTO.html

13. Vogt H, Buse K, Hesse H and Kratzig E, 2001. Growth and holographic characterization of nonstoichiometric sillenite-type crystals. J. Appl. Phys. 90: 3167-3173.

14. Marrucci L, 2008. Generation of helical modes of light by spin-to-orbital angular momentum conversion in inhomogeneous liquid crystals. Mol. Cryst. Liq. Cryst. 488: 148-162.

15. Karimi E, Piccirillo B, Nagali E, Marrucci L and Santamato E, 2009. Efficient generation and sorting of orbital angular momentum eigenmodes of light by thermally tuned q-plates. Appl. Phys. Lett. 94: 231124.

16. Piccirillo B, D'Ambrosio V, Slussarenko S, Marrucci L and Santamato E, 2010. Photon spinto-orbital angular momentum conversion via an electrically tunable q-plate. Appl. Phys. Lett. 97: 241104 .

17. Marrucci L, Manzo C and Paparo D, 2006. Optical spin-to-orbital angular momentum conversion in inhomogeneous anisotropic media. Phys. Rev. Lett. 96: 163905.

18. Beth R A, 1936. Mechanical detection and measurement of the angular momentum of light. Phys. Rev. 50: 115-125.

19. Vasylkiv Yu, Skab I and Vlokh R, 2011. Measurements of piezooptic coefficients $\pi_{14}$ and $\pi_{25}$ in $\mathrm{Pb}_{5} \mathrm{Ge}_{3} \mathrm{O}_{11}$ crystals using torsion induced optical vortex. Ukr. J. Phys. Opt. 12: 101-108.

Vasylkiv Yu., Krupych O., Skab I. and Vlokh R., 2011. On the spin-to-orbit momentum conversion operated by electric field in optically active $\mathrm{Bi}_{12} \mathrm{GeO}_{20}$ crystals. Ukr.J.Phys.Opt. 12: $171-179$.

Анотація. В роботі експериментально та теоретично досліджено перетворення оптичного спінового кутового моменту в орбітальний кутовий момент (СКМ-ОКМ), яке виникало в кристалах $\mathrm{Bi}_{12} \mathrm{GeO}_{20}$ під дією зовнішнього конічного електричного поля, завдяки ефекту Покельса. Виявлено виникнення „бубликової” моди і оптичного вихора в системі, яка складалась з право обертаючого ииркулярного поляризатора, зразка підданого дії конічного електричного поля i ліво обертаючого циркулярного аналізатора. Виявлено, що присутність природної оптичної активності приводить до суттєвого зменшення ефективності СКМ-ОКМ перетворення. 